Aus dem Pathologisch-anatomischen Institut der Universität in Leipzig. (Direktor: Geh. Rat Marchand.)

\title{
Zur pathologischen Anatomie der akuten gelben Leberatrophie.
}

Von Priv.-Doz. Dr. med. et phil. Carly Seyfarth.

Wie die zahlreichen Veröffentlichungen über akute gelbe Leberatrophie der letzten Jahre zeigen, kommt diese Erkrankung jetzt zweifellos häufiger zur Beobachtung als früher. Die gleiche zunehmende Häufigkeit sahen wir im Pathologischen Institut der Universität Leipzig. Vor dem Kriege kam hier durchschnittlich jährlich ein Fall zur Sektion. Seit 1915 bis jetzt wurden 29 Fälle seziert.

Ueber die pathologisch-anatomischen Grundlagen dieser Erkrankung gehen die Ansichten in den Veröffentlichungen der letzten Jahre recht auseinander. Meines Erachtens führt besonders eine Vermengung der anatomisch und auch klinisch gut abgrenzbaren Stadien der akuten gelben Leberatrophie zu Irrtümern. Eine solche Einteilung schlug ich auf dem Pathologentag 1921 vor. Sie ergibt sich ganz zwanglos 1. nach der Krankheitsdauer und 2. nach dem jeweiligen pathologisch-anatomischen Befunde. Wir müssen unterscheiden: 1. eine akute, 2. eine subakute, 3 . eine subchronische, 4. eine chronische Form der akuten gelben Leberatrophie.

Die akute Form der Leberatrophie bekommen wir nur sehr selten bei der Sektion zu Gesicht. Nur diese Form verdient wirklich den Namen akute gelbe Leberatrophie, denn nur in diesem Stadium hat das Organ eine wirklich gelbe oder bräunlichgelbe Farbe. Die zu Lebzeiten unmittelbar nach Krankheitsbeginn vergrößerte Leber wird bei der Sektion innerhalb der ersten 4-5 Tage nach Beginn der Erkrankung als verkleinertes Organ von schlaffer, weicher Beschaffenheit mit scharfen Rändern und runzeliger Kapsel gefunden. Mik roskopisch ist der völlige Zerfall der Leberzellen charakteristisch. Dieser beginnt am häufigsten in der Peripherie der Läppchen. Die Zentra sind zumeist noch als gut erhalten zu erkennen. Durch diese Tatsache läßt sich der plötzlich auftretende, schwere Ikterus zwanglos erklären: Der völlige Zerfall in der Peripherie der Leberläppchen zerstört die hier befindlichen Gallenkapillaren. $\mathrm{Er}$ verhindert so den AbfluB der Galle. Diese tritt in die Lymphwege und damit in die allgemeine Blutbahn über und führt so den Ikterus herbei.

Viel häufiger kommt die subakute Form zur Sektion. Im Zeitraum vom 5. Erkrankungstage bis zum Ende der 3. Woche wird eine Leber gefunden, die keine einheitlich gelbe Farbe mehr hat. Je nach der Dauer der Erkrankung treten an der gelben Oberfläche bzw. dem Durchschnitt rote Streifen und Flecke auf, die immer mehr zunehmen und der Leber das bekannte rot und gelb gefleckte, bunte Aussehen geben. Jetzt ist die Konsistenz der Leber nicht mehr schlaff und weich, sondern $z a ̈ h, z a ̈ h-e l a s t i s c h$, zum Teil auffallend derb, je nachdem die roten atrophischen oder die gelben, erhaltenen, wenn auch zum Teil veränderten Abschnitte überwiegen. Mikroskopisch entsprechen die roten Stellen dem Bilde des völligen Schwundes der Leberbälkchen. Allenthalben sind reparatorische Vorgänge, Gallengangswucherungen und Neubildungen von Leberzellen aus diesen und aus erhaltengebliebenen Leberzellen zu sehen. Aszites ist in diesem subakuten Stadium nicht häufig.

Im subchronischen Stadium (4. Woche bis 8. Monat nach Krankheitsanfang) hat die Wiederherstellung einen viel höheren Grad er-

1) Schweiz. m. W. 1921 S. $440 .-^{2}$ ) D. Arch. f. klin. M. 130 S. 342 u. 132 S. $358 .-$ 3) Briefliche Mitteilung. 
reicht. Es ist zu einer multiplen knotigen Hyperplasie gekommen. Aut der Oberfläche und dem Durchschnitt der verkleinerten Leber ist das neugebildete Lebergewebe in Form von kirsch- bis walnußgroßen, kugeligen Vorwölbungen zu erkennen, deren Farbe rötlichgelb und deren Konsistenz ziemlich weich ist, weicher jedenfalls als das dazwischenliegende, je nach dem Blutgehalt blaßbis dinnkelrote, scheinbar eingesunkene Lebergewebe. Dieses rote Gewebe besteht aus sehr gefäBreichem Bindegewebe mit reichlichen zelligen Infiltraten. Regenerationsvorgänge sind mikroskopisch allenthalben zu verfolgen. In einzelnen seltenen Fällen konnten wir neben dieser knotigen Hyperplasie ein Auftreten von frischen parenchymatösen Degenerătionen, also ein Wiederaufflackern des ganzen Vorganges nachweisen, das jetzt wahrscheinlich zım Tode führte.

Aszites gehört bei dieser subchronischen Form der akuten gelben Leberatrophie zur Regel. Alle hierhergehörigen Fälle zeigten fast ohne. Ausnahme größere Aszitesmengen, die zwischen 1 und 10 Litern schwankten. Einige Autoren, vor allem Umber, weisen immer wieder darauf hin, daß Aszites bei unkomplizierten Fällen von akuter gelber Leberatrophie nich t vorkäme. Dem ist nicht so. Dieser Irrtum konnte nur dadurch entstehen, da $B$ eben die verschiedenen Formen der akuten gelbeı Leberatrophie nicht auseinandergehalten wurde11. - Was die Entstehung des Aszites anbelangt, so läßt er sich unschwer erklären: Die mit den fortsch reitenden reparatorischen Vorgängen immer zunehmenden interstitiellen Bindegewebswucherungen innerhalb und vor allem in der Periplerie der Azini bedingen ein Zugrundegehen der Pfortaderkapillaren und so einen Widerstand in der Blutzirkulation. Hierdurch kommt es im Pfortaderkreislauf zu Stauung und damit zu Aszites.

Bei der chronischen Form werden stark verkleinerte Lebern gefunden, deren Ober- und Unterfläche vollständig höckrig ist und kleinere und größere Knoten aus Lebergewebe zeigen. Zwischen diesen finden sich strahlige bindegewebige Einziehungen. Falls eben keine wiederholten stärkeren Schübe der parenchymatösen Degeneration auftreten, können die Wiederherstellungsbestrebungen immer weiter fortschreiten. So kann sich uns bei der Sektion in der Zeit von 9 Monaten bis Jahre nach der 11rsprünglichen Erkrankung ein Bild bieten, das nach völliger Ausheilung von einer großlappigen Zirrhose nicht mehr zu unterscheiden ist Aber auch noch in diesem Stadiun kann es zu einem Wiederaıfflackern des ganzen Vorganges, zil erneutem Zerfall kommen. Es sind dies jene mehrfach beschriebenen Fälle, in denen sich angeblich die akute gelbe Leberatrophie an eine sich langsam entwickelnde, bis dahin unbemerkte Leberzirrhose angeschlossen haben soll. RieB möchte solche Fälle als sekundäre (komplizierte) Leberatrophie bezeichnen. Derartige Fälle von akuter gelber Leberatrophie als Schlußstein einer Leberzirrhose oder einer Syphilis hepatis können ja wohl vorkommen. Immerhin glaube ich, $\mathrm{da} \beta$ es sich bei den meisten so gedeuteten Fällen um einfache chronische, gewissermaßen rezidivierende Formen einer ursprïnglich akuten gelben Leberatrophie handelt. Freilich wird der diagnostische Nachweis dieses Vorgangs, vor allem bei schleichendem Verlauf der Erkrankung, sehr schwer sein. In einem unserer chronischen Fälle, in dem eine solche der großlappigen Zirrhose nahestehende Leber gefunden wurde, konnte das Hervorgehen aus einem früheren Stadium der akıten gelben Leberatrophie unmittelbar nacligewiesen werden, und zwar 1. aus dem genauen Krankenbericht und 2. dadurch, daß ein Chirurg bei einer Probelaparatomie die Leber in diesem früheren Stadium, 8 Monate vor der Sektion, zu Gesicht bekommen hatte. In dieser Weise, indem sich allmählich völlig das Krankheitsbild einer Leberzirrhose entwickelt, kantr es zu einer Ausheilung kommen.

Heilungen der akıtten gelben Leberatrophie, die Ausgänge in großlappige Zirrhosen, kommen häufiger vor, als man bisher annahm. Es ist wahrscheinlich, daß vor allem vielen derartigen, bei $\mathrm{Kind}$ e $\mathrm{n}$ gefundenen Leberzirrhosen eine früher durchgemachte akute gelbe Leberatrophie zugrundeliegt. Im Kindesalter sind ja die subakuten 1111d subchronischen Stadien der akitten gelben Leberatrophie und vor allem mildere, abortive Form nicht sehr selten. Wegerle stellte 191439 Erkrankungen im Kindesalter zusammen, und P la u t konnte in einer Leipziger Dissertation 192061 Fälle von akuter gelber Leberatrophie im Kindesalter zilsammenbringen. Unter unseren 29 Fällen der letzten Jahre befanden sich 5 Individuen im Alter von $1-13$ Jahren.

Zur Aetiologie der akuten gelben Leberatrophie möchte ich Folgendes anführen: Es ist mir aufgefallen, daß die Häufung unserer Fälle in die Jahre 11 m ittelbar nach der Heimkehr unserer Soldaten von den Kriegsschanplätzen fiel. Wir sahen in den Kriegsjahren 1914-1918 im ganzen 7, in den Jahren 1919-1921 zusammen 22 Fälle. Ganz entsprechend sind andere Beobachtungen, z. B. die von $\mathrm{L} 1 \mathrm{~b}$ arsch oder die von Umber. Dieser beobachtete in Berlin-Westend 1912-1919 3 Fälle, 19197 Fälle, 19207 Fälle. 19214 Fälle bis März! Im Felde bzw. in den Militärspitälern wurde während des Krieges auffallend viel akute gelbe Leberatrophie geselien. Benda macht auf eine solche Häufinng aufmerksam, und Hart schreibt: ,Im Felde hat man sie bei den Soldaten öfters gesehen, auch in der Heimat betraf die Mehrzahl Männer im besten Mannesalter, die teils selbst Soldaten waren, teils zweifellos vielfach mit solchen in Berührung kamen." Herxheimer sezierte 7 Soldaten. die an akuter gelber Leberatrophie gestorben waren. Franz $M$ iille r erlebte während des Krieges eine solche Häufung von Leberatrophiefällen itı den Ingolstädter Lazaretten, daß man sie als eine
Epidemie bezeichnen kann. Von 21 Fällen schweren Ikterıs, die in einem halben Jahr vorkamen, starben 13 an akuter gelber Leberatropliie. Ein Unterarzt, der sich bei der Sektion eines dieser Fälle verletzt hatte, starb 6 Wochen danach in Erlangen an akuter gelber Leberatrophie. Dieser Fall ist besonders genau untersucht nnd begutachtet worden. Syphilis war. nicht vorhandent.

Umber nimmt an, die jetzige Zunahme hänge zusammen nit der qualitativen Verschlechterung der Kostverhältnisse, wie sie die Not der Zeit in den unteren Bevölkerungsschichten mit sich bringe. Hierdurch sollen die gastro-intestinalen Katarrhe und dadurch die Infekte der Gallenwege zunehmen. Dem kann ich nicht bedingungslos beistimmen, denı dann müßte der Höhepunkt der Zunahme bei der Zivilbevölkerıng in die Kriegshungerjahre 1916 ind 1917 fallen, und das trifft nach unseren Erfahrungen nicht zu. 'Dann wäre mir schon wahrscheinlicher die Häl1fung in den Jahren 1919 und 1920 durch Einschleppen der „Erreger" durch heimkehrende Leichterkrankte usw. zu erklären.

Doch das ist eine Hypothese. Als einwandfrei feststehend ist anzunehmen, daß die akute gelbe Leberatrophie ätiologisch $k$ ein $\mathrm{e}$ einheitliche Krankheit ist: 1. kann die Krankheitsbereitschaft der Leber zu dieser Erkrankung durch ganz verschiedene Momente bedingt sein; 2. kann die Erkrankung durch verschiedene Ursachen a usgelöst werden. Zweifellos haben sich die wichtigsten disponierenden Bedingungen unmittelbar nach dem Weltkriege vermehrt: Syphilis, Aborte, Allgemeininfektionen, Nahrungsverschlechterung.

Auf eine dieser krankheitsbereitenden Ursachen, die Syphilis, wird in den Veröffentlichungen immer wieder hingewiesen. Fast alle Ingolstädter Fälle und die von $\mathrm{Herxheimer}$ mitgeteilten schließen sich unmittelbar an Frühstadien der Syphilis an. Nel1erdings glaubt E. Ma ye r (B. kl. W. 1921, Nr. 31) auf Grund seiner Beobachtungen an einem großen Sektionsmaterial annehmen zu können, daß die Syphilis als konstanter Faktor in der Aetiologie der akuten gelben Leberatrophie zil betrachten sei. Dem ist nach unseren Beobachtungen nicht so. Von unseren 29 Fällen, die 9 Männer, 15 Frauen und 5 Kinder betrafen, war nur ein ganz kleiner Teil, 4 mit Syphilis in Zissammenhang zu bringen. Nach den Krankengeschichten war zumeist anf Syphilis genau gefahndet worden. Einer unserer 4 ,Synhilisfälle" ist insofern besonders interessant, als er sich an das Frühstadium einer Syphilis anschloß, die nachgewiesenermaßen bis dahin in keiner Weise weder mit Hg-Präparaten noch mit Salvarsan behandelt war. Ein Beweis, daß energische $\mathrm{Hg}$ - oder Salvarsankıren nicht unbedingt bei den Syphilisfällen als auslösende Faktoren für eine akute gelbe Leberatrophie in Betracht kommen müssen, wie manche Autoren glauben.

Die eigentlichen a uslösenden Ursachen der zıım Zerfall des Leberparenchyms führenden autolytischen Vorgänge können 1 . toxi * sche und 2. infektiöse sein. Ganz zweifellos gibt es Gifte, die von außen eingebracht oder auch endogen, vor allem enterogen cntstanden, eine akute gelbe Leberatrophie erzeugen. Näheres hierüber wissen wir jedoch immer noch nicht. Einer unserer Fälle gehört ätiologisch insofern hierher, als die Erkranking an akuter gelber Leberatrophie unmittelbar im Anschluß an eine Bandwurmkur begann. Für einen anderen Teil der Fälle kommen aber sicher belebte Krankheitserreger in Betracht. Umber möchte dabei dem. Infekt der Gallenwege vom Darm her die Hauptrolle zuschreiben, andere halten auch eine Infektion auf dem Blut- und Lymphwege für möglich. Fränkel nimmt an, daß dabei die Krankheitserreger selbst sehr verschieden sein können. Ob dies richtig ist, oder ob der $\mathrm{Er}$ reger ein einheitlicher ist, vielleicht eine Spirochätenart, in Analogie zum Icterus infectiosus und zum tropischen Gelbfieber, ist noch völlig unbestimmt. Eine endgültige Entscheidung können nur weitere bikteriologische und experimentelle Forschungen bringen.

Für die letzteren erscheint mir eine Tatsache besonders wichtig. die von bisherigen Untersuchern nicht beachtet worden ist, da $B$ nämlich auch Haustiere (Hund, Schwein, Rind) an akuter gelber Leberatrophie spontan erkranken können. Joest sezierte vor kurzem auch einen Bären des Zoologischen Gartens in Dresden, der plötzlich an akuter gelter Leberatrophie erkrankt und gestorben war. Die mikroskopischen Veränderungen bei der tierischen akuten gelben Leberatrophie sind denen der menschlichen völlig gleich. Auch die vier von mir aufgestellten Formen finden wir bei ihr. Wir haben zunächst den akuten Zerfall, dann die Gelb- und Rotfleckung. Weiterhin im subchronischen Stadium die knotige Hyperplasie und bei der Wiederherstellung auch den Ausgang der akuten gelben Leberatrophie in eine knotige, großlappige Leberzirrhose, die beim Hund in seltenen Fällen gefunden wird und die auf eine durchgemachte, frühere akute gelbe Leberatrophie hindeutet. Wie beim Menschen, tritt beim Hund die Erkrankung auf, zumeist ohne daß eine bestimmte Ursache nachzilweisen ist. Sicher ist auch bei den Tieren die Aetiologie keine einheitliche. In manchen Fällen kommen endogen bzw. enterogen entstandene Gifte in Betracht, so werden z. B. Lupinenvergiftungen (Lupinosen) als auslösende Ursachen angesprochen, in anderen 'Fällen Intoxikation durch verdorbene Nahrungsmittel. In den meisten Fällen tritt aber die Erkrankung bei den Tieren ganz plötzlich auf, ohne daB eine bestimmte Ursache nachzuweisen ist.

Literaturi siehe C. Seyfarth, Akute gelbe Leberatronhie. Verh. D. path. 